\title{
Comportamiento de muertes neonatales ocurridas en el Nuevo Hospital Monte España, del 1 de enero 2010 al 31 de diciembre 2011
}

Dr. Léster Benjamín Pichardo Mejía

dr.pichardomejia@hotmail.com, lester.pichardo@hospitalmontespana.com.ni

\section{RESUMEN}

El presente estudio, tiene como objetivo determinar el comportamiento de las muertes neonatales ocurridas en el NUEVO HOSPITAL MONTE ESPAÑA del 1 de enero 2010 al 31 diciembre 2011. Entre los principales resultados obtenidos, tenemos que en el año 2010 hubo 13 muertes neonatales de las cuales 10 eran neonatales temprana, mientras que en el año 2011 ocurrieron 9 muertes neonatales perteneciendo 7 al grupo de mortalidad neonatal. En cuanto al grupo etáreo más frecuente de las madres en que se presentaron los casos de mortalidad neonatal temprana fue el de 20 a 34 años con $96 \%$ de los casos. Los antecedentes personales patológicos y no patológicos fueron negativos en el $100 \%$ de las pacientes en estudio. Con respecto a los factores de riesgo maternos de los recién nacido que sufrieron mortalidad neonatal temprana el $41.2 \%$ eran Bigestas, el $23.5 \%$ Primigestas, con un período intergenésico mayor de 2 años en el $52.9 \%$ de los casos, se captaron la mayoría en el I trimestre con $58.8 \%$, se realizaron 4 o más controles en un $70.6 \%$ de los casos y todas las pacientes se clasificaron como Embarazos de Alto Riesgo Obstétrico (ARO). Los recién nacidos en estudio predominaron en el grupo gestacional de 27 a $36.6 \mathrm{SG}$ con $64.7 \%$, seguido del grupo de 37 a $41.6 \mathrm{SG}$ con $23.5 \%$ y el grupo de 20 a $26.6 \mathrm{SG}$ con $11.8 \%$. El sexo predominante de los neonatos en estudio predominaron los masculinos con 70.6. La vía de nacimiento que predominó fue la abdominal con $52.9 \%$. El Apgar al minuto de nacido en la mayoría de los neonatos es de 4-7 con 47.1\%, seguido de 8-10pts con $35.3 \%$, y de $0-3$ pts con $17.6 \%$. A los 5 minutos la mayoría obtuvo un puntaje de 8-10pts con $70.6 \%$, seguido de $4-7$ pts con $17.6 \%$ y $0-3$ pts con $11.8 \%$.

Palabras claves: muerte neonatal, primigesta, factores de riesgos, Hospital Monte España.

\section{INTRODUCCIÓN}

El conocimiento de la mortalidad perinatal es un hecho de gran importancia porque proporciona una imagen del nivel de desarrollo y calidad de los pueblos. La tasa de mortalidad perinatal es un indicador que resume el riesgo de morir del feto y recién nacido como consecuencia del proceso reproductivo.

La Organización Mundial de Salud (OMS) define la mortalidad neonatal como la muerte producida entre el nacimiento (niño que después de la expulsión completa de la madre, independientemente de la edad gestacional, respire o de alguna señal de vida) hasta los 28 días de vida. Comprendiendo la mortalidad neonatal precoz hasta los 7 días de vida y la tardía de los 8 a 28 días.

Durante este período, el producto de la concepción está sometido a una serie de riesgos que dependen fundamentalmente del ambiente exterior que puede ser tan favorable o no como el ambiente materno.

Los cambios fisiológicos que implica para el feto, el paso de la vida intrauterina a la extrauterina, determina la presencia de patologías propias del período perinatal siendo consecuencia de la alteración de alguno de los mecanismos de adaptación. Durante este período, el producto de la concepción está sometido a una serie de riesgos preconcepcionales, del embarazo y parto que determinan la sobrevida del recién nacido o la morbimortalidad perinatal.

En los últimos 20 años, la tasa de mortalidad infantil en Latinoamérica ha disminuido en 50\%. Sin embargo, la mortalidad neonatal ha quedado casi inalterable. Nacen muertos en el mundo alrededor de 4,3 millones de niños y 3,3 millones mueren en la primera semana de vida, de estos 7,6 millones de muertes perinatales, el $98 \%$ ocurren en países en vías de desarrollo.

En Nicaragua la mortalidad perinatal constituye un problema de salud pública asociada a múltiples 
factores socioeconómicos, culturales y educacionales, un sistema de información insuficiente que no permite obtener la información necesaria para el registro y análisis de los casos.

El Nuevo Hospital Monte España es un hospital privado que presta servicios de salud al Instituto Nicaragüense de Seguridad Social, desde hace 19 años aproximadamente, enfocado al Programa EnfermedadMaternidad y los Programas de alto costo (Oncología y Hemodiálisis). En el Nuevo Hospital Monte España, durante el año 2010 ocurrieron 13 muertes neonatales de las cuales 10 eran neonatales temprana y en el año 2011 ocurrieron 9 muertes neonatales perteneciendo 7 al grupo de mortalidad neonatal temprana.

A pesar de los avances de la atención en neonatología y las estrategias del cuidado perinatal, la mortalidad neonatal continúa siendo un problema, fundamentalmente en los países subdesarrollados como el nuestro. Por lo tanto considero necesario explorar las características de riesgo de los recién nacidos en esta unidad hospitalaria.

Con el presente estudio, se pretende analizar el comportamiento de la mortalidad neonatal que ocurrió en estos dos años con el objetivo de elaborar planes de mejoras para disminuir este indicador de mortalidad y mejorar la calidad de la atención en los derechohabientes adscritos a esta IPSS.

\section{MATERIALES Y MÉTODOS}

a. Tipo de estudio: Estudio Descriptivo, retrospectivo de corte transversal.

b. Población de estudio: Todos los recién nacidos ingresados al Servicio de Neonatología del Nuevo Hospital Monte España del 1 de enero del 2010 al 31 de diciembre del 2011.

c. Muestra: Todos los casos de mortalidad neonatal temprana sucedidos en el servicio de Neonatología del Nuevo Hospital Monte España del 1 de enero del 2010 al 31 de diciembre del 2011, que totalizan 17 casos.

\section{d. Criterios de selección:}

\section{Criterios de inclusión}

- Todos los casos de mortalidad neonatal temprana del Servicio de Neonatología del Nuevo Hospital Monte España del 1 de enero del 2010 al 31 de diciembre del 2011.

- Hijos de madres adscritas al Instituto Nicaragüense de Seguridad Social y afiliadas al Nuevo Hospital Monte España.
- Hijos de madres cuyos controles prenatales se realizaron en el Nuevo Hospital Monte España.

\section{Criterios de exclusión:}

- Recién nacidos egresados vivos del Servicio de Neonatología del Nuevo Hospital Monte España del 1 de enero del 2010 al 31 de diciembre del 2011.

- Recién nacidos hijos de madres con atención privada en la institución.

- Casos de mortalidad neonatal tardía.

\section{Criterios de selección de expedientes:}

- Se tomaron todos los expedientes de casos de mortalidad neonatal temprana debidamente registrados en área de Estadísticas del Hospital

Monte España en el período del 1 de enero del 2010 al 31 de diciembre de 2011.

- Expedientes de las madres que se encontraban en per cápita y en área de Admisión del Hospital.

- Expedientes de madres cuyos controles prenatales se realizaron en el hospital, en el período en estudio.

e. Unidad de análisis: Expedientes clínicos de casos de mortalidad neonatal temprana del Servicio de Neonatología y los expedientes de las madres en el período del evento en estudio.

\section{RESULTADOS}

La tasa de mortalidad neonatal precoz en esta institución durante el período estudiado fue de $10.6 \%$ en 2010 y de $7.0 \%$ en 2011. Los casos de mortalidad neonatal precoz fue mayor en el año 2010 con 58.8\%, seguido del año 2011 con $41.2 \%$. (Ver Tabla n $\left.{ }^{\circ} 1.\right)$

La edad materna fue más representativa en el grupo etáreo de 20 a 24 años con $94 \%$, seguido del grupo etáreo de 35 a 49 años con $6 \%$. En cuanto a la procedencia la gran mayoría son del área urbana con $94 \%$, seguido del área rural con 6\%. La escolaridad más representativa es la secundaria con $47.1 \%$, seguido de universitaria con $29.4 \%$ y primaria con $23.5 \%$. En cuanto al estado civil el $65 \%$ corresponde a las casadas, seguido del $29 \%$ las acompañadas y el $6 \%$ las solteras. (Ver Tabla $\mathrm{n}^{\mathbf{0}} 2$ ).

Los antecedentes personales patológicos y no patológicos fueron negativos en el $100 \%$ de las pacientes en estudio. En cuanto a los antecedentes Gineco-obstétricos, el $41.2 \%$ son Bigestas, el 23.5\% son Primigestas, el $17.6 \%$ Trigestas y el $17.6 \%$ Multigestas, es importante señalar que el resto de antecedentes fueron negativos, ejemplo cesárea anterior, aborto, entre otros. (Ve Tabla ${ }^{\circ} 3$ ). 
Tabla n¹. Tasa de mortalidad neonatal precoz en el Hospital Monte España. Periodo 2010 - 2011

\begin{tabular}{|c|c|c|c|c|}
\hline \multirow{2}{*}{ AÑO } & \multirow{2}{*}{} & \multicolumn{3}{|c|}{ MORTALIDAD NEONATAL PRECOZ } \\
\cline { 2 - 5 } & NACIDOS VIVOS & $\mathbf{N}^{\circ}$ & $\%$ & TASA (X 1000NV) \\
\hline $\mathbf{2 0 1 0}$ & 935 & 10 & 58.8 & 10.6 \\
\hline $\mathbf{2 0 1 1}$ & 995 & 7 & 41.2 & 7.0 \\
\hline Total & 1930 & 17 & 100.0 & 8.8 \\
\hline
\end{tabular}

Fuente. Registros estadísticos del Hospital Monte España

Tabla $n^{\circ} 2$. Características socio-demográficas de las progenitoras de los recién nacidos en estudio. Periodo $2010-$ 2011

Edad $n=17$

\begin{tabular}{|c|c|c|}
\hline Edad & $n^{\circ}$ & $\%$ \\
\hline 15 - 19 años & 0 & 0 \\
\hline 20 - 34 años & 16 & 94 \\
\hline $35-49$ años & 1 & 6 \\
\hline Total & 17 & 100 \\
\hline \multicolumn{3}{|c|}{ Procedencia $n=17$} \\
\hline Rural & 1 & 6 \\
\hline Urbano & 16 & 94 \\
\hline Total & 17 & 100 \\
\hline \multicolumn{3}{|c|}{ Escolaridad } \\
\hline Primaria & 4 & 23.5 \\
\hline Secundaria & 8 & 47.1 \\
\hline Universitaria & 5 & 29.4 \\
\hline Analfabeta & 0 & 0.0 \\
\hline Total & 17 & 100.0 \\
\hline \multicolumn{3}{|c|}{ Estado civil } \\
\hline Soltera & 1 & 6 \\
\hline Casada & 11 & 65 \\
\hline
\end{tabular}


Comportamiento de muertes neonatales ocurridas en el Nuevo Hospital Monte España... pág. 49-55

\begin{tabular}{|l|c|c|} 
Acompañada & 5 & 29 \\
\hline Divorciada & 0 & 0 \\
\hline Viuda & 0 & 0 \\
\hline Total & 17 & 100 \\
\hline
\end{tabular}

Fuente. Expedientes clínicos de las pacientes en estudio atendidas en Hospital Monte España. Periodo 2010 -

2011

Tabla $n^{\circ} 3$. Antecedentes gineco-obstétricos de las pacientes en estudio. Periodo 2010 - 2011

\begin{tabular}{|c|c|c|}
\hline \multirow{2}{*}{ Antecedentes } & \multicolumn{2}{|c|}{ MORTALIDAD NEONATAL PRECOZ } \\
\hline & $n^{\circ}$ & $\%$ \\
\hline Primigesta & 4 & 23.5 \\
\hline \begin{tabular}{|l|} 
Bigesta \\
\end{tabular} & 7 & 41.2 \\
\hline Trigesta & 3 & 17.6 \\
\hline Multigesta & 3 & 17.6 \\
\hline Total & 17 & 100 \\
\hline
\end{tabular}

Fuente. Expedientes clínicos de las pacientes en estudio atendidas en Hospital Monte España. Periodo 2010 -

2011

El período intergenésico en la mayoría de las pacientes es mayor de 2 años con $52.9 \%$, seguido por menor de 2 años con $23.5 \%$ y las que no aplican por ser Primigestas con $23.5 \%$. El trimestre de captación de las pacientes predomino en el I trimestre con $58.8 \%$, seguido del II trimestre con $29.4 \%$ y el III trimestre con $11.8 \%$. En cuanto al número de controles prenatales predominó aquellas con 4 o más con $70.6 \%$, seguido de 1 a 3 controles con $17.6 \%$ y sigue aquellas con ningún control con $11.8 \%$. La clasificación del embarazo el $100 \%$ son pacientes con embarazos de Alto riesgo (ARO).

Las patologías durante el embarazo predominaron aquellas clasificadas en el grupo de Otros con 58.8\%, las infecciones de vías urinarias con $52.9 \%$, seguido de las infecciones cervicovaginales con $41.2 \%$, luego la amenaza de aborto con $29.4 \%$, la amenaza de parto pretérmino con $23.5 \%$, Polihidramnios y Preclampsia en mismo porcentaje con $17.6 \%$ y los Oligohidramnios y la anemia en mismo porcentaje con $5.9 \%$.

Los recién nacidos en estudio predominaron en el grupo gestacional de 27 a $36.6 \mathrm{SG}$ con $64.7 \%$, seguido del grupo de 37 a $41.6 \mathrm{SG}$ con $23.5 \%$ y el grupo de 20 a 26.6 SG con $11.8 \%$. El sexo predominante de los neonatos en estudio predominaron los masculinos con $70.6 \%$, seguido de las femeninas con $23.5 \%$ y sexo indeterminado con $5.9 \%$. La vía de nacimiento predomino la abdominal con $52.9 \%$ seguida de la vaginal con $47.1 \%$. En cuanto al peso al nacimiento predomino los de $1000-2499 \mathrm{gr}$ con $47.1 \%$, seguido de $2500-3999$ gr con $29.4 \%$ y de $500-999$ gr con $23.5 \%$.

El Apgar al minuto de nacido en la mayoría de los neonatos es de 4-7 pts. con 47.1\%, seguido de 8-10 pts.con $35.3 \%$, y de $0-3$ pts. con $17.6 \%$. A los 5 minutos la mayoría obtuvo un puntaje de $8-10$ pts. con $70.6 \%$, seguido de $4-7$ pts. con $17.6 \%$ y $0-3$ pts. con $11.8 \%$. El período de supervivencia más representativo fue de 1-3 días con $64.7 \%$, seguido de $23.5 \%$ en menor de 1 día y $11.8 \%$ aquellos sobrevivientes de 4-7 días.

Entre las patologías de los neonatos más frecuentes sobresalió el Síndrome de dificultad respiratoria (SDR) con $52.9 \%$, la Sepsis neonatal temprana con $29.4 \%$, las malformaciones congénitas con $17.6 \%$. Luego aquellas agrupadas como Otro con 17.6\%, la 
asfixia moderada y severa en mismo porcentaje con $11.8 \%$ y enfermedad de membrana hialina con $5.1 \%$.

Las principales causas de mortalidad en los neonatos en estudio son en la mayoría síndrome de dificultad respiratoria y sepsis neonatal temprana con $29.4 \%$, respectivamente; seguido por malformaciones congénitas con $17.6 \%$, luego por asfixia severa con $11.8 \%$, y la enfermedad de membrana hialina y broncoaspiración con 5.9\%, respectivamente.

En cuanto al peso de los neonatos según los días de supervivencia, en el grupo que sobrevivió menos de 1 día: el $75 \%$ fueron aquellos con peso de $1000-2499 \mathrm{gr}$, seguido de $25 \%$ en aquellos de 2500-3999gr. El grupo que sobrevivió de 1-3 días predominó aquellos con peso de $1000-2499 \mathrm{gr}$ y $2500-3999 \mathrm{gr}$ con $36.4 \%$ cada uno, y $27.3 \%$ los de 500-999gr. El grupo que sobrevivió de 4-7 días predominaron aquellos con peso de 500-999gr y 1000-2499gr con 50\% cada uno.

\section{DISCUSIÓN}

Se analizó el total de defunciones neonatales precoz de los años 2010 y 2011, siendo en total 17 casos, de 1930 nacimientos, con una tasa de 10.6 y 7.0 en 2010 y 2011, respectivamente. Se considera que son muertes que pueden ser prevenibles e intervenibles con el acceso oportuno y la calidad de los servicios de atención materna y perinatal.

En el presente estudio se encontró que el grupo etáreo de mayor representación es el de 20 a 34 años, corresponde con un estudio de una Empresa prestadora de salud en Colombia 2007 cuyo número mayor de casos de mortalidad perinatal se encontró en este grupo de edades; contrasta con las estadísticas nacionales y la literatura que reportan a las edades extremas como factores de riesgo de mortalidad perinatal. Se considera que la explicación guarda relación al tipo de población que se atiende en este hospital, la procedencia urbana de la mayoría de las madres es acorde a las localizaciones de las empresas laborales y en contraste con los reportes del MINSA y ENDESA 06/07 donde las de origen rural son asociadas a la mayoría de casos de mortalidad perinatal.

En este estudio la escolaridad de las pacientes es muy buena siendo en su mayoría de secundaria y universitaria, además son embarazos de madres casadas o acompañadas. Contrario a lo que se expresa en un estudio sobre Factores asociados a la mortalidad perinatal por Ochoa en Juigalpa, donde la mayoría de las madres de casos en estudio tenían un bajo nivel de escolaridad por debajo del sexto grado (77.3\%) y según ENDESA 2006/07 la mortalidad alta se encuentra en los niños de mujeres "sin educación".

Los antecedentes patológicos personales y no personales en este estudio fueron negativos en el total de los casos incluidos, contrario a lo expresado por un estudio realizado por Delgado en Colombia, 2007, es el cual el tabaquismo, alcoholismo, aspectos psicológicos y candidiasis vaginal representaron asociación de riesgo a los casos de mortalidad perinatal.

Entre los antecedentes gineco-obstétricos más sobresalientes es la paridad: en su mayoría Bigestas y Primigestas quienes según la literatura tienen una alta correlación con bajo peso al nacer y mortalidad neonatal. Estos resultados corresponde parcialmente con un estudio realizado en Hospital Bertha Calderón Roque en el año 2004 sobre Morbi-Mortalidad perinatal, donde se muestra que las Primigestas y las Multigestas son las más afectadas en los casos estudiados. En otro estudio sobre factores asociados a la mortalidad perinatal en Juigalpa, 2002, que también refiere las Primigestas y gran multíparas como factor de riesgo. En cuanto al resto de antecedentes ginecoobstétricos resultaron negativos en este estudio.

El período intergenésico mayor de 2 años predominó en este estudio, señalando que este factor no es determinante como riesgo en este estudio, tal como sucedió en el estudio de Factores de Riesgo Asociados a Mortalidad Neonatal en Hospital Fernando Vélez Paíz, 2009-2010.

En cuanto al trimestre de captación predominó en el I trimestre con más de la mitad de las pacientes del estudio, con 4 o más controles prenatales en todo el embarazo, seguido de 1 a 3 controles, siendo clasificadas como pacientes con embarazos de Alto riesgo (ARO) el total de las pacientes en estudio. Contrario a lo ocurrido en un estudio realizado en El Hospital Carlos Marx, 1991, en el cual el Tercer Trimestre alcanzó más frecuencia de captación de los embarazos, y a lo encontrado por otros autores quienes concluyen que a menor número de consultas prenatales mayor es el riesgo de mortalidad neonatal, hasta 6 veces más alta sobre todo en las mujeres sin ninguna consulta prenatal. 
Los patologías durante el embarazo predominaron las infecciones de vías urinarias seguido de las infecciones cervicovaginales, la amenaza de aborto y la amenaza de parto pretérmino. Esto en coincidencia con otros estudios sobre Factores de Riesgo Asociados a Mortalidad Neonatal, donde se muestra predominó de las infecciones: cervicovaginitis e infecciones de vías urinarias y las amenazas de parto pretérmino, y según referencia de la literatura que los relaciona como factores de riesgo predominantes en los casos de mortalidad perinatal.

La edad gestacional al momento de nacimiento predominante en este estudio fue mayores de 27 semanas con pesos mayores de 1000gr, según Osorio y Romero encontraron hasta 55 veces más riesgo de uerte perinatal en los pretérminos y de acuerdo a la literatura que refiere la prematurez con bajo peso al nacer como una asociación con la mortalidad perinatal; el sexo predominante fueron los masculinos, nacieron a través de cesáreas, según Covarrubias y Col, en su estudio encuentran el hecho de haber nacido por cesárea como un factor protector en relación con la mortalidad. Siendo controversial dicho tema ya que no se ha podido poner de manifiesto la cesárea con descenso de mortalidad perinatal.

El Apgar al minuto de nacido en la mayoría de los neonatos es mayor de 4 pts. y a los 5 minutos de 8-10 pts., los casos de 0-3 pts. son una minoría, sin embargo esto no ha sido un factor protector para estos casos.

Según la literatura a menor puntaje mayor riesgo, sin embargo el nivel de secuelas por falta de oxigenación no debe medirse por el puntaje recibido sino por las características clínicas y en asociación con los factores de riesgo: pretérmino y bajo peso al nacer (menor de $2500 \mathrm{gr}$ ) potencializan incluso más de 50 veces el riesgo de mortalidad.

Entre las patologías de los neonatos asociadas a la mortalidad más frecuente sobresalió el Síndrome de dificultad respiratoria (SDR), la Sepsis neonatal temprana y las malformaciones congénitas. Esto corresponde con la literatura y estudios que describen estos factores con una mayor asociación y alto valor predictivo para mortalidad perinatal.

En cuanto los días de supervivencia según el peso de los neonatos, el grupo que sobrevivió 1 a 3 días representó la mayoría en los 3 grupos de peso en estudio, sin embargo en este estudio el peso no fue un factor protector, pues la mortalidad en período menor a 1 días fue mayor en aquellos mayores de 1000gr.

\section{CONCLUSIONES}

De los factores socio-demográficos más sobresalientes en este estudio son la edad materna de 20-34 años, procedentes del área urbana, con escolaridad entre secundaria y universitaria y estado civil casada o acompañadas.

Los factores de riesgo maternos asociado a mortalidad fetal son las Bigestas seguido de las Primigestas, todas cursaban con embarazos de alto riesgo; en su gran mayoría el período intergenésico mayor de 2 años, la captación en el I trimestre y los 4 o más controles prenatales no representaron un factor protector en este estudio tal como lo refiere la literatura y estudios de referencia.

Las patologías durante el embarazo más frecuentes en los casos estudiados son las infecciones de vías urinarias, seguido de las infecciones cervicovaginales, la amenaza de aborto y la amenaza de parto acorde a las patologías con mayor asociación de riesgo según la literatura y estadísticas nacionales, a excepción de la Preeclampsia que no se presentó en estos casos.

Los recién nacidos en estudio cursaban una edad gestacional predominante en el grupo de 27 a $36.6 \mathrm{SG}$ seguido del grupo de 37 a $41.6 \mathrm{SG}$.

El sexo de los recién nacidos prevalecieron los masculinos, la mayoría nació por vía abdominal con peso al nacimiento de 1000-2499gr seguido de 25003999gr. Con Apgar más frecuente mayor de 4 pts., la asfixia severa no se mostró dominante como factor de riesgo de mortalidad.

Entre las patologías más frecuentes sobresalió el Síndrome de dificultad respiratoria (SDR), la Sepsis neonatal temprana y las malformaciones, con un período de supervivencia más frecuente de 1-3 días seguido de aquellos con período menor de 1 día, donde el peso no se mostró como un factor protector, ya que la mayoría de fallecimiento en menos de 1 día se presentó en los de 1000gr o más.

\section{BIBLIOGRAFÍA}

American College of Obstetrician and Gynecologist. Educational Bulletin. Special Problems of Multiple Gestation, 1998 Compendium of Selected Publications. American College of Obstetricians and Gynecologists 2002. 
Aylward, Glen. Perinatal Asphyxia: Effects of Biological and Environmental Risks, Clinics In Perinatology, June 1993.

Burrow G, Ferris T. Complicaciones médicas durante el embarazo: Manejo obstétrico de la paciente de alto riesgo. Editorial Panamericana, cuarta edición.

Cabero, L. et al. Obstetricia y Medicina Materno Fetal. Buenos Aires; Madrid: Medica Panamericana, 2007.

Cabero, R. Riesgo elevado obstétrico. Masson SA. $1^{\text {a }}$ edición. 1996. PP 21-22.

Centro Latinoamericano de Perinatología y desarrollo humano. Atención inmediata al recién nacido. Publicación científica $\mathrm{N}^{\circ}$ 378-2001. CLAP. OPS (Consulado 3 May 2010). Disponible en: www.clap.ops.oms.org/web_2005/BOLETINE S\%20Y\%20NOVEDADES/BOLETIN17.pdf

Cobo E. Metrorragias de la segunda mitad de la gestación, desprendimiento prematuro de placenta normoinserta, placenta previa. Segunda Edición, Santiago de Chile 1994.
Delgado, A. Et al. Mortalidad perinatal de la población afiliada a una EPS de Pasto. Departamento de Nariño. 2007

Díaz, F. Factores asociados a la mortalidad perinatal, neonatología, HBCR. Enero - Diciembre, 2008. Tesis para obtener el título de especialista en Gineco-Obstetricia. HBCR

Freetts, R. Schmittdiel, J. Mclean FH, Usher RH,

Goldman MB.Increased maternal age and the risk of fetal death.N Engl J Med. 1995.

Gabbe, S. et al. Obstetricia, normalidad y complicaciones en el embarazo: Incidencia, epidemiologia y etiología del RCIU 2002. Editorial Marban, 3ra.Edición.

Greenwood R, Samms-Vaughan JG, Ashley D. Past obstetric history nad risk of perinatal death. Pediatric and Perinatal Epidemiology, 1994.

Lede R. Evaluación clínica de la hipoxia fetal en el embarazo. Capítulo 3, "Del sufrimiento fetal al daño cerebral” Urman J. y Sebastiani M. 1995-

Martínez, C. Factores de Riesgo Asociados a Mortalidad Neonatal en la UCIN del HMIFVP Enero 2009 a Noviembre 2010. 